\title{
Huge values of room-temperature dielectric constants in pellets of poly(3-methylthiophene)
}

\author{
S. Moraes and L. Walmsley ${ }^{\text {a) }}$ \\ Departamento de Física, Instituto de Geociências e Ciências Exatas, Universidade Estadual Paulista, \\ Caixa Postal 178, CEP 13500-970, Rio Claro, S.P., Brazil \\ E.C. Pereira and A.A. Correa \\ Departamento de Química, Universidade Federal de São Carlos, Caixa Postal 676, CEP 13560-970, \\ São Carlos, S.P., Brazil
}

(Received 4 November 2003; accepted 16 March 2004)

Room temperature data of impedance and phase angle in pellets of electrochemically synthesized $\mathrm{ClO}_{4}^{-}$doped poly(3-methylthiophene) (P3MT) were analyzed assuming the sample being represented by a parallel resistor-capacitor (RC) circuit or by a series RC circuit. The last assumption proved to be the correct one, and to confirm it we use the sample as the RC component of a resistor-capacitor-inductor series resonator. We discuss the possibility of this RC series behavior to be due to a charge-density wave characteristic also evidenced from the huge values of the low-frequency dielectric constant of the system.

\section{INTRODUCTION}

Conducting polymers are materials of increasing importance for the fabrication of electronic and optoelectronic devices. Conducting polymers exhibit novel properties not typically available in other materials. These novel properties make possible a number of applications including polymer light-emitting diodes (LEDs), conducting polymers as electrochemical materials, polymer photodetectors, and polymer photovoltaic cells. ${ }^{1}$

Among the conducting polymers, the polythiophene derivative poly(3-methylthiophene) (P3MT) has been used with great versatility in a number of applications. The electrochemical synthesis of this polymer allows endless possibilities due to great number of variables that can be changed in the synthesis process, giving rise to polymers with different properties. According to Tourillon, ${ }^{2}$ responsible for the first electrochemical synthesis, ${ }^{3}$ the working electrode can be $\mathrm{Pt}, \mathrm{Au}$, or glass coated with $\mathrm{SnO}_{2}$ or $\mathrm{In}_{2} \mathrm{O}_{3}$. A tin-oxide (TO) electrode has also been used. ${ }^{4}$ The electrolytic medium typically consists of an organic solvent like acetonitrile, dichloromethane, or benzoitrile. The supporting electrolyte is in general a salt of $\mathrm{BF}_{4}^{-}, \mathrm{ClO}_{4}^{-}, \mathrm{PF}_{6}^{-}$, or $\mathrm{SO}_{3} \mathrm{CF}_{3}^{-}$. The films can be grafted on the electrode with either a controlled potential or a controlled current. The molarity of the monomer and the temperature are also synthesis variables giving different results concerning the electrical properties. ${ }^{5}$ The

\footnotetext{
a) Address all correspondence to this author.

e-mail: walmsley@rc.unesp.br

DOI: 10.1557/JMR.2004.0260
}

oxidized polymer can be reversibly reduced. In the reduced form, P3MT was used in field-effect devices. ${ }^{6}$ In the oxidized state, it was used as a solar cell in a P3MT/CuInSe ${ }_{2}$ junction. ${ }^{7}$ In a recent article, ${ }^{8}$ we have shown room-temperature weak ferromagnetic behavior in pellets of partially reduced P3MT. We have also shown evidence of metamagnetic behavior in the same system.

The stability of P3MT at ambient conditions is a subject far from being clarified. According to Tourillon, ${ }^{2}$ it is stable in the oxidized and reduced forms at ambient conditions. But the influence of light, oxygen, ${ }^{6}$ and moisture on the P3MT properties has been already observed, although the real role played by these agents in the material is not understood. We have observed that partially doped pellets are not stable at ambient conditions. The electric properties are affected (decrease in the conductivity) as well as the magnetic properties (change from ferromagnetic to diamagnetic behavior), ${ }^{9}$ evidencing the decrease of the polarons with charge $e$ and spin $1 / 2$. The $\mathrm{ClO}_{4}{ }^{-}$doped pellets described in this work, on the other hand, seem to be much more stable at ambient conditions. Keeping the sample in the dark at ambient conditions, we have only observed a reversible dependence on moisture (increase in the conductivity). The same stability at ambient conditions was not observed for $\mathrm{BF}_{4}{ }^{-}$ doped pellets. This observation is in agreement with the strong interaction of $\mathrm{ClO}_{4}{ }^{-}$ions and the polymer in oxidized poly(3-methylthiophene). ${ }^{10}$

Most of the low-frequency ( $\leqslant 1 \mathrm{MHz}$ ) dielectric constant measurements in conducting polymers have been performed in films ${ }^{11}$ with values of dielectric constants around $10^{3}$. But low values (less than $10^{3}$ ) were also 


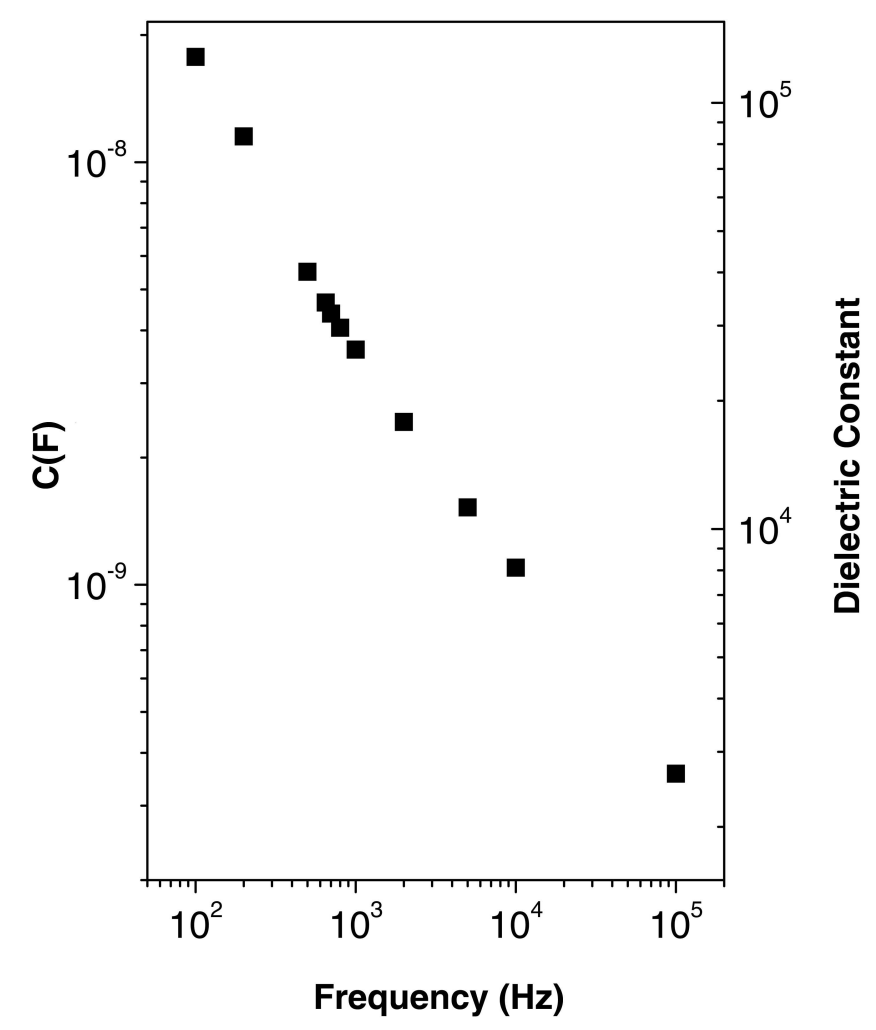

FIG. 1. Room-temperature values of capacitance (left) and dielectric constant (right) versus frequency (log-log scale) under the assumption that sample behaves as a RC parallel circuit.

observed in pellets. ${ }^{12}$ The use of the cavity perturbation technique to measure microwave dielectric constants ${ }^{13}$ has shown in turn high values of microwave dielectric constants $\left(\geqslant 10^{4}\right)$.

In this work, we report the measurement of roomtemperature impedance $(\mathrm{Z})$ and phase angle $(\delta)$ in the range from $100 \mathrm{~Hz}$ to $1 \mathrm{MHz}$ in pellets of doped poly(3methylthiophene). The values of dielectric constant and resistivity were obtained from the $\mathrm{Z}$ and $\delta$ values using two different assumptions: (i) the sample being represented by a parallel RC circuit, and (ii) the sample being represented by a series RC circuit. Although more unusual, we will show in this work that the second assumption is the correct one. To prove this assumption, we use the sample as the RC component of a resistor-capacitorinductor (RCL) series resonator.

\section{EXPERIMENTAL}

P3MT was electrochemically synthesized. at $5{ }^{\circ} \mathrm{C}$ on a Pt electrode by a constant potential of $1.48 \mathrm{~V}$ in an acetonitrile solution with $0.1 \mathrm{M}$ methylthiophene and $0.1 \mathrm{M}$ $\mathrm{LiClO}_{4}$. The powder deposited on the electrodes was removed and uniaxially pressed $(6.2 \mathrm{kbar})$ to make pellets of radius $r=2 \mathrm{~mm}$ and thickness $\mathrm{d}=405 \mu \mathrm{m}$. The room-temperature values of the impedance and the phase angle were obtained using a Solartron (England) 1260 Impedance Analyzer with the silver painted sample fixed by mechanical clamping between two gold recovered electrodes in a capacitive setup. The RCL series circuits, with known $\mathrm{L}$ and small resistance $(2 \Omega)$, used the same amplitude value for the ac voltage signal as the Solartron measurements. The ac current was measured with a Minipa (Brazil) ET-2051 A microammeter.

\section{RESULTS}

Figure 1 shows the values of capacitance (left) and dielectric constant (right) versus frequency obtained from the $\mathrm{Z}$ and $\delta$ data on the assumption that the sample behaves as a RC parallel circuit. Figure 2 shows the values of capacitance (left) and dielectric constant (right) versus frequency on the assumption that the sample behaves as a RC series circuit. Figure 3 shows for both assumptions, RC parallel (open triangles) and RC series (full triangles), the values of resistance (left) and for RC series the values of resistivity (right) versus frequency. From Fig. 2, we can observe that a series RC circuit will imply huge values of dielectric constants. On the other hand, a RC parallel circuit will imply high values of dielectric constants (Fig. 1).

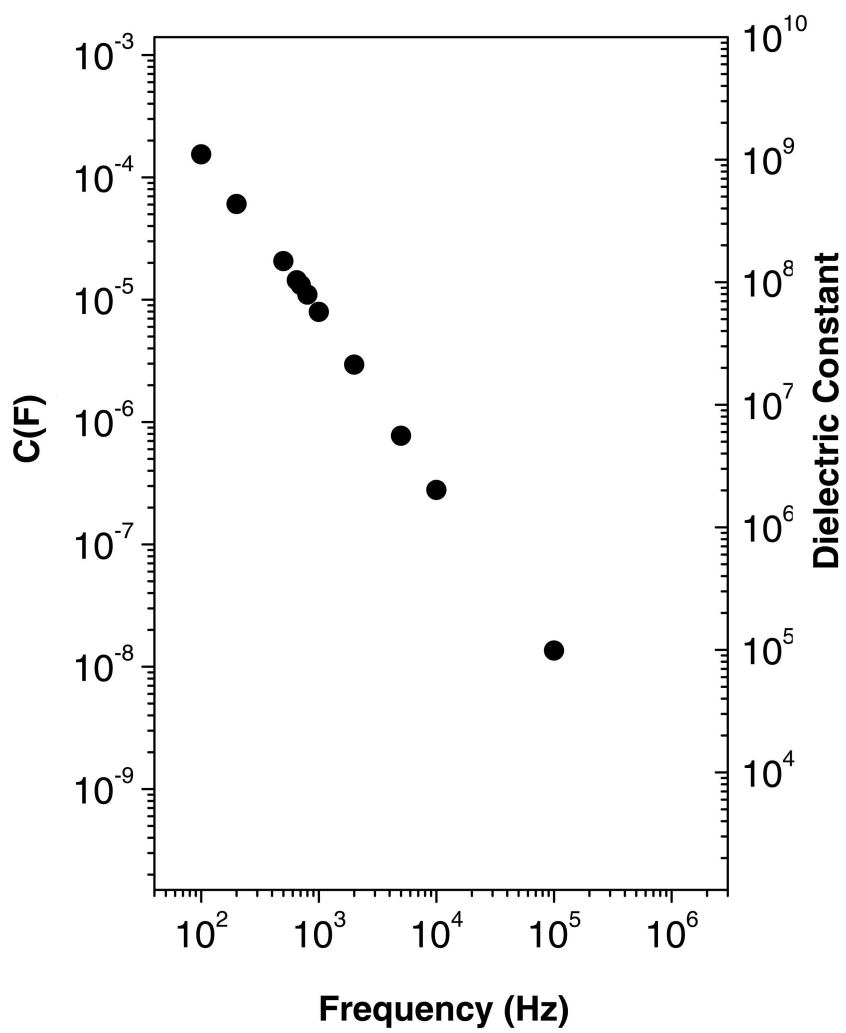

FIG. 2. Room-temperature values of capacitance (left) and dielectric constant (right) versus frequency (log-log scale) under the assumption that the sample behaves as a RC series circuit. 
To check both hypotheses, we tried to see a minimum in the current arranging the sample with known inductors in a RCL parallel circuit or to see a maximum in the current for a RCL series arrangement. The second option proved to be the correct one, and the values of $\mathrm{I}^{2}$ versus frequency are shown in Fig. 4. The data obtained from the RCL series measurement are summarized in Table I.

The value of the resonance frequency was confirmed using the modulus of the reactance values obtained from the Solartron 1260 impedance data and is shown in Fig. 5. The capacitive reactance of the sample was obtained assuming the RC series circuit. The inductive reactance of the inductor was obtained assuming a RL series circuit. The frequency for which both reactance modulus data coincide is the same frequency at which the RCL series resonator shows a current maximum in Fig. 4. We can also observe very good agreement of the capacitance and resistance values shown in Table I with those for the corresponding frequency $(\mathrm{C}=13.2 \mu \mathrm{F}$ and $\mathrm{R}=943 \Omega$ ) shown in Figs. 2 and 3.

A possible explanation for this RC series-like behavior and the huge values of dielectric constant in pellets of doped P3MT could be a charge-density wave characteristic. It is well established that in charge-density wave

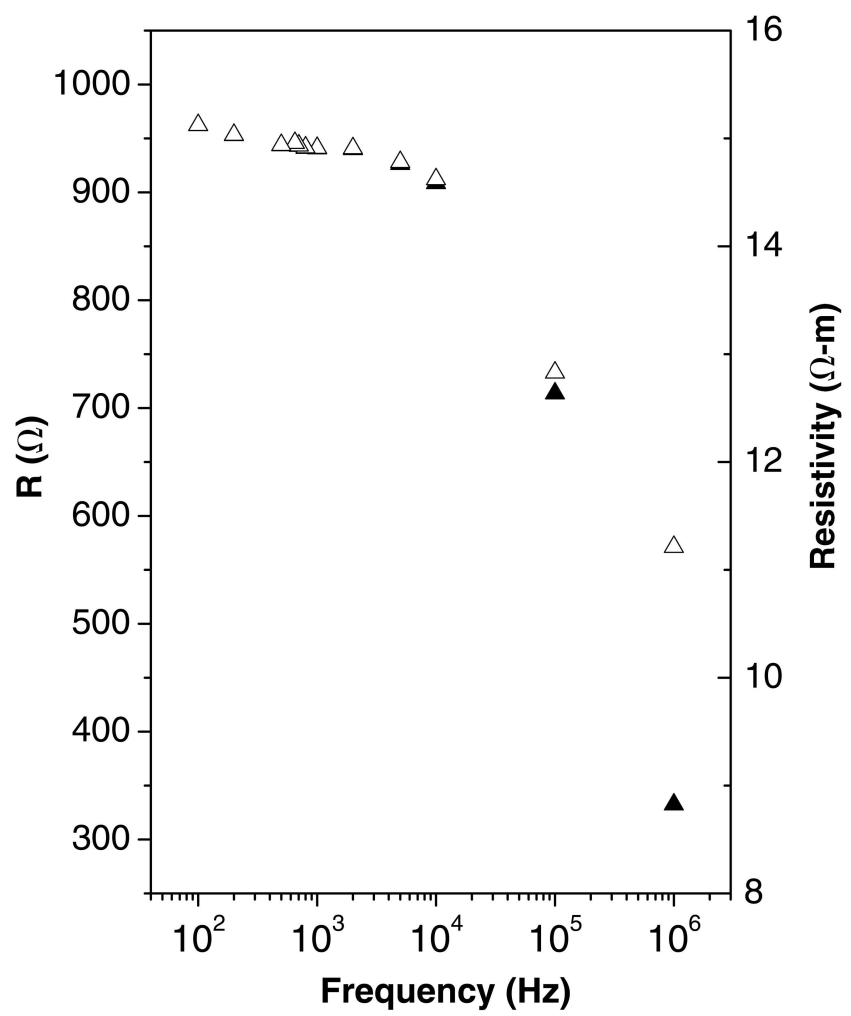

FIG. 3. $(\triangle)$ Room-temperature values of resistance versus frequency (log scale) under the assumption that the sample behaves as a RC parallel circuit. ( $\mathbf{\Delta})$ Room-temperature values of resistance (left) and resistivity (right) versus frequency (log scale) under the assumption that the sample behaves as a RC series circuit.

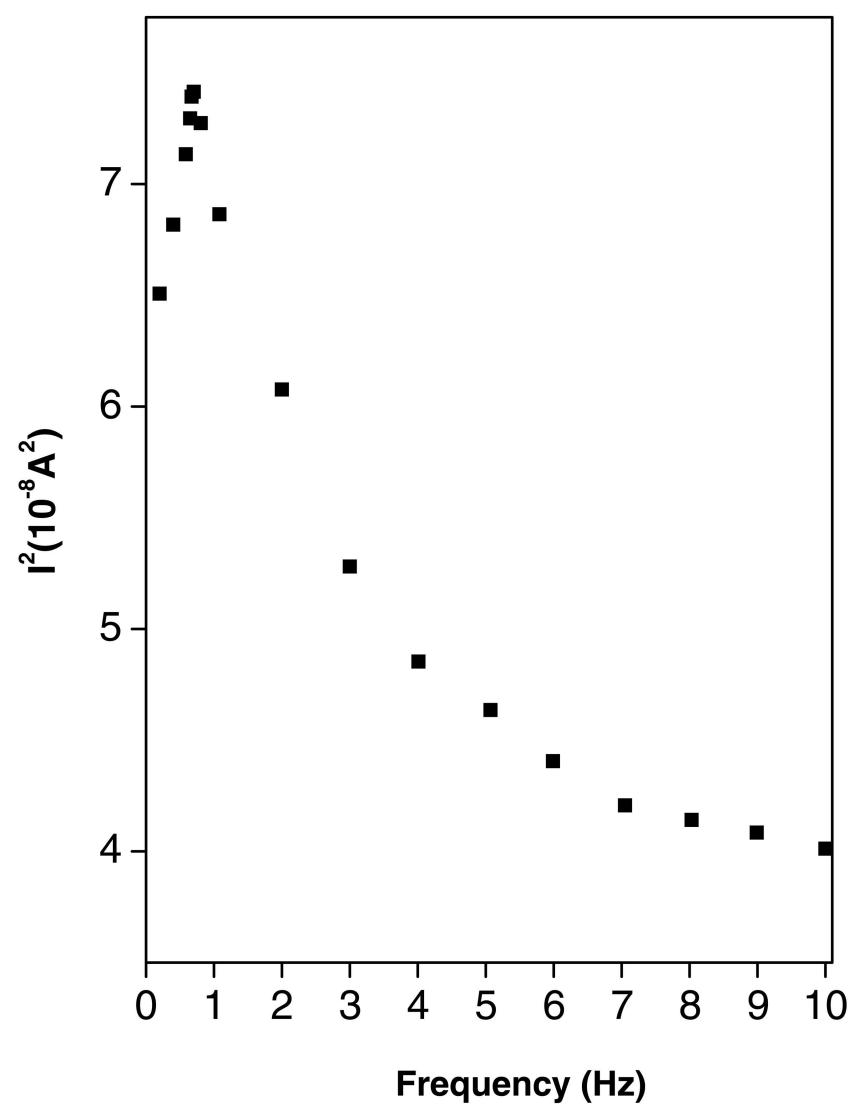

FIG. 4. Room-temperature values of $\mathrm{I}^{2}$ versus frequency in a RCL series circuit.

TABLE I. Parameters obtained from the RCL series circuit with a known inductor.

\begin{tabular}{lcccc}
\hline \hline Temperature & Inductor & $\begin{array}{c}\text { Resonance } \\
\text { frequency }\end{array}$ & Capacitance & Resistance \\
\hline $294 \mathrm{~K}$ & $3.62 \mathrm{mH}$ & $702 \mathrm{~Hz}$ & $14.2 \mu \mathrm{F}$ & $1.1 \mathrm{k} \Omega$ \\
\hline \hline
\end{tabular}

(CDW) systems, there is a dipole moment associated with the phase mode, and huge values of dielectric constant have been measured at low frequencies in conventional charge-density wave materials like $\mathrm{NbSe}_{3}$ and $\mathrm{TaS}_{3} .{ }^{14}$ The CDW system was modeled ${ }^{15}$ as a resistance due to normal electrons in parallel with the CDW condensate contribution that can be represented by a resistance in series with a capacitance. The normal electrons contribute to a frequency-independent conductivity. If the conductivity due to normal electrons is small, the resistance is high and can be neglected compared to the $\mathrm{CDW}$ contribution. The system will behave as a RC series circuit. Other evidences of room-temperature $\mathrm{CDW}$ behavior in pellets of P3MT have already been discussed, such as field-dependent conductivity for very low electric fields. ${ }^{16}$ 


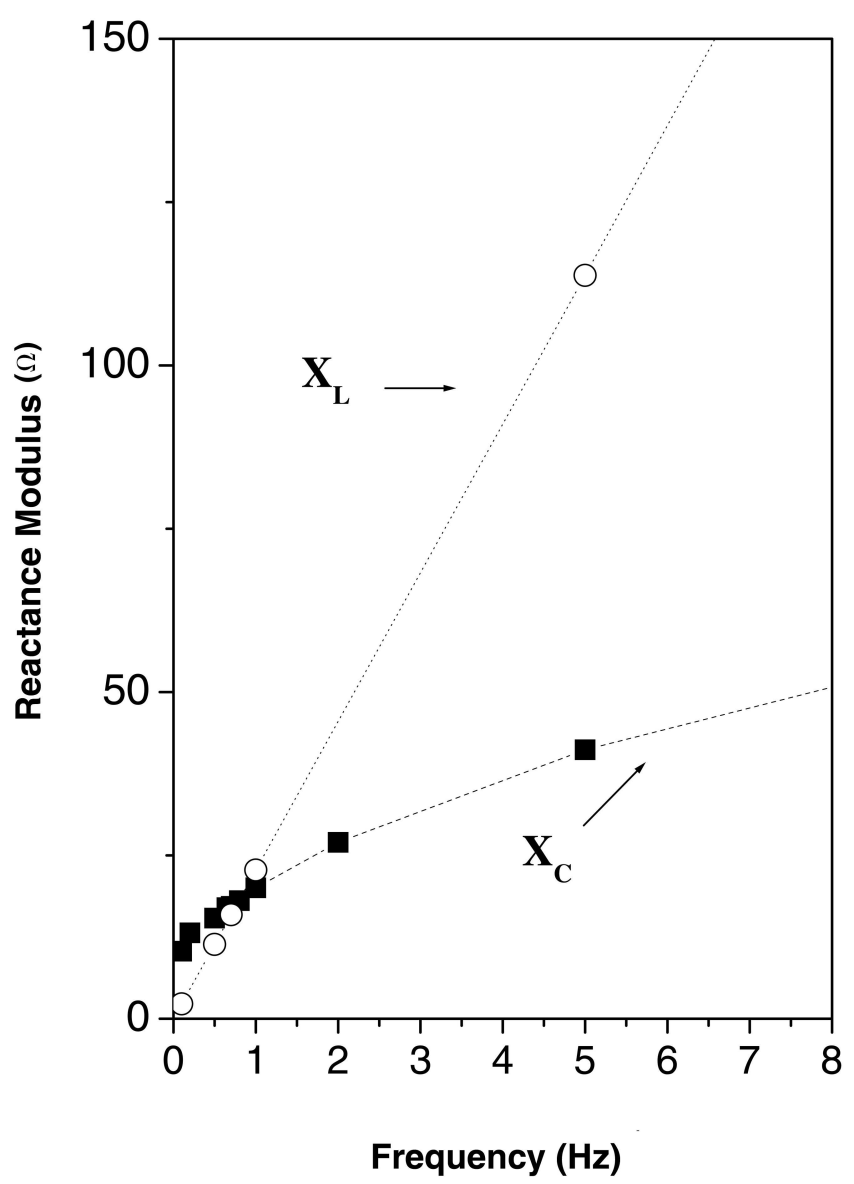

FIG. 5. Room-temperature reactance modulus versus frequency. The capacitive reactance values were obtained under the assumption that the sample behaves as a RC series circuit. The inductive reactance values were obtained under the assumption that the inductor behaves as a RL series circuit.

\section{CONCLUSIONS}

The behavior of the pellet of $\mathrm{ClO}_{4}^{-}$doped poly(3methylthiophene) as a RC series-like component of a RCL series resonator revealed a huge value for the lowfrequency dielectric constant. A possible explanation for both the RC series-like behavior and the huge low-frequency dielectric constant could be a CDW behavior. If the CDW characteristic is confirmed, the system could find an application in electronic devices. A room-temperature "Josephson like" junction could use the $\mathrm{CDW}$-conducting polymer system as the condensate instead of using the superconductor as the condensate. For the metal-CDW-metal junction, some theoretical calculations have already appeared in the literature. ${ }^{17,18}$

\section{ACKNOWLEDGMENTS}

We are in debt to Dr. G.A. Santarine for the technical support. This work was partially supported by Fundação de Amparo à Pesquisa do Estado de São Paulo, Conselho Nacional de Desenvolvimento Científico e Tecnológico e Fundação Para o Desenvolvimento da Unesp.

\section{REFERENCES}

1. A.J. Heeger: (Nobel Lecture) Rev. Mod. Phys. 73, 681 (2001).

2. G. Tourillon: Handbook of Conducting Polymers, Vol. 1, edited by T.A. Skotheim (Marcel Dekker Inc., New York, 1986).

3. G. Tourillon and F. Garnier: J. Electroanal. Chem. 135, 173 (1982).

4. R. Valaski, A.F. Bozza, L. Micaroni, and I.A. Hümmelgen: J. Solid State Electrochem. 4, 390 (2000).

5. M. Sato, T. Susumo, and K. Kaeriyama: Synth. Met. 14, 279 (1986).

6. D.M. Taylor, H.L. Gomes, A.E. Underhill, S. Edge, and P.I. Clemenson: J. Phys. D: Appl. Phys. 24, 2032 (1991).

7. P.J. Sebastian, S.A. Gamboa, M.E. Calixto, H. Nguyen-Cong, P. Chartier, and R. Perez: Semicond. Sci. Technol. 13, 1459 (1998).

8. O.R. Nascimento, A.J.A. de Oliveira, A.A. Correa, L.O.S. Bulhões, E.C. Pereira, V.M. Souza, and L. Walmsley: Phys. Rev. B 67, $144422(2003)$.

9. E.C. Pereira, A.A. Correa, L.O.S. Bulhões, P.C. Aleixo, J.A. Nóbrega, A.J.A. de Oliveira, W.A. Ortiz, and L. Walmsley: J. Magn. Magn. Mater. 226, 2023 (2001).

10. F-J. Pern and A.J. Frank: J. Electrochem. Soc. 137, 2769 (1990).

11. Z.H. Wang, E.M. Scherr, A.G. MacDiarmid, and A.J. Epstein: Phys. Rev. B 45, 4190 (1992).

12. V. Luthra, R. Singh, and A. Mansigh: Synth. Met. 119, 291 (2001).

13. J. Joo, S.M. Long, J.P. Pouget, E.J. Oh, A.G. MacDiarmid, and A.J. Epstein: Phys. Rev. B 57, 9567 (1998).

14. G. Grüner: Rev. Mod. Phys. 60, 1129 (1988).

15. G. Grüner, L.C. Tippie, W.G. Clark, and N.P. Ong: Phys. Rev. Lett. 45, 935 (1980).

16. V.M. Souza, L. Walmsley, A.A. Correa, and E.C. Pereira: Solid State Commun. 126, 141 (2003).

17. M.I. Visscher and G.E.W. Bauer: Phys. Rev. B 54, 2798 (1996).

18. L.E. Oxman, E.R. Mucciolo, and I.V. Krive: Phys. Rev. B 61, $4603(2000)$. 